\title{
Double detonation drivers for a shock tube/tunnel
}

\section{CHEN Hong, FENG Heng \&YU Hongru}

High Temperature Gas Dynamics Laboratory, Institute of Mechanics, Chinese Academy of Sciences Beijing 100080, China Correspondence should be addressed to Chen Hong (email: hongchen@imech.ac.cn) Received February 23, 2004

\begin{abstract}
ARecent progress on detonation drivers is reviewed. Performances of the forward detonation driver and backward detonation driver have been observed. To eliminate occurrence of a Taylor wave following the detonation wave in the primary driver and to improve the performance of the detonation driver, an additional backward detonation driver was proposed to attach to the end of the forward detonation driver. When the ratio of the initial pressures between the additional and the primary drivers becomes larger than or equal to a critical value, the Taylor wave will disappear, and thus a homogeneous driving gas with high pressure and high temperature can be generated. Furthermore, an over-driving detonation wave will be also obtained, which can increase the driving capability.

Keywords: gaseous det onati on, shock tube, shock tunnel.

DOI : 10. 1360/03yw0305
\end{abstract}

Shock tubes have been used extensively in many fields of aerodynamics, gaseous chemical reactions, high-temperature gas kinetics, and explosive phenomena. A shock tube is an efficient laboratorial device used to generate controllable shock waves, which can compress and accelerate the tested gas to attain high pressure, high temperature and high velocity. When the gas is accelerated, the local acoustic velocity increases, accompanied by rising temperature. As a result, Mach numbers of the gas flow are limited. An expansion nozzle connected to the end part of the shock tube can break this limit. This kind of equipment is named shock tunnel.

In order to acquire an intense shock wave, the driving gas in the driver section should have high acoustic velocity and high pressure. The detonation driver is an economical and effective driver. A driving gas of high acoustic velocity and high pressure is easily generated by a detonation wave in the detonation driver, which initiates an intense shock wave. There are two operating detonation modes in the detonation driver. The first is the backward detonation driver. Igniter for the detonation initiation is placed near 
the main diaphragm, and the generated detonation waves will propagate upstream toward the end of the driver. The second is the forward detonation driver, in which the detonation wave is initiated at the end of the driver section, and propagation direction of the detonation waves is the same as that of the incident shock wave.

$\mathrm{Yu}^{[1]}$, Lee ${ }^{[2]}$, and Gier and Jones ${ }^{[3]}$ have carried out detonation driver experiments successfully. Their experimental results showed that the attenuation ratio of the incident shock wave produced by a backward detonation driver was lower than that by the forward detonation driver. The results exhibited good repeatability. In the experiments reported by $\mathrm{Yu}$, the initial pressure of detonable gases was the highest and the shock tube exhibited violent vibration during the experimental process ${ }^{[1]}$. This vibration was caused by the abrupt pressure of the reflection wave acting on the end wall of the tube when the denotation wave also arrived at the end of the tube. The amplitude of the abrupt pressure was one hundred times larger than the initial pressure ${ }^{[4]}$. Resolution of this safety factor is required for the backward detonation driver to be practically used.

Balcarzak and Johnson ${ }^{[5]}$ investigated several characteristics of the forward detonation driver. They concluded that the forward detonation driver was unacceptable for aerodynamic testing because the shock waves generated by the forward detonation driver attenuate both with length and with time of shock wave propagation. The attenuation effect was generated by a rarefaction wave (Taylor wave) following the detonation wave and overtaking the incident shock wave. In ref. [6] the additonal driver utilized hydrogen as the driver gas attached to the upstream end of the detonation driver. When the diaphragm between the additional and the primary drivers ruptured, the hydrogen in the additional driver expanded and accelerated, generating a high-speed moving gas column. The gas column then compressed the detonating gas in the primary driver and an intense shock wave formed. The intense shock wave could initiate detonation directly, the Taylor wave and the attenuation of the incident shock wave generated by Taylor wave would be eliminated if the velocity of the gas column could be equal to or larger than that of the gas behind the wave front. However, the pressure of the hydrogen was not high enough, and consequently the velocity of the expanded hydrogen was below that of the detonation gas and initiation of detonation was difficult to accomplish upon rupture of the diaphragm. Thus, the experiment failed.

In the 1980s, the test-flight results from American shuttlecraft indicated that effects of real gases strongly influenced the characteristics of aerodynamic force on the lifting body. Moreover, under the demand of supersonic combustion testing, ground-based experiments simulating high-enthalpy conditions are urgently needed. Yu and colleagues ${ }^{[7,81}$ proposed connecting a dumping section to the end of the driving section to eliminate the reflected high pressure of the detonation wave. Accordingly, the backward detonation driver can generate test gases with high enthalpy and high stagnation pressure. Through comprehensive analysis and comparison of various kinds of strong detonation drivers, Bakos et al. ${ }^{[9]}$ concluded: "In view of benefit-cost ratio, the detonation driver is antici- 
pated to be the optimum choice for current supersonic research." Subsequently, detonation drivers have moved into a new stage of development.

\section{Characteristics of forward and backward detonation drivers}

1.1 Flow field structure behind the detonation wave initiated at the end wall of a closed tube

Gas detonation experiments have revealed that the structure of the flow field is very complicated because the quasi-periodic concussion of the detonation wave is coupled with a series of transverse waves propagated through the detonation wave front. However, the period and the amplitude of the detonation concussion, as well as associated interference, are much lower than characteristic parameters of the detonation driver and the idealized Chapman-Jouguet (CJ) detonation model meets the analytical requirements for characteristics of the detonation driver. This paper is based on that model.

Taylor ${ }^{[10]}$ and Zel'dovich ${ }^{[11]}$ determined that the flow field occurred behind detonation wave initiated at the end wall of the closed tube and that the field is composed of constant-speed detonation waves and following self-simulating rarefaction waves. Fig. 1 shows distributions of pressure, density, temperature, and velocity of the gas following the detonation wave. The symbols $u_{0}, x$, and $t$ represent the propagation velocity, distance, and time of the detonation wave, respectively. Penetrating the rarefaction wave, the detonation gas is finally decelerated to form a stationary and thermodynamic-state

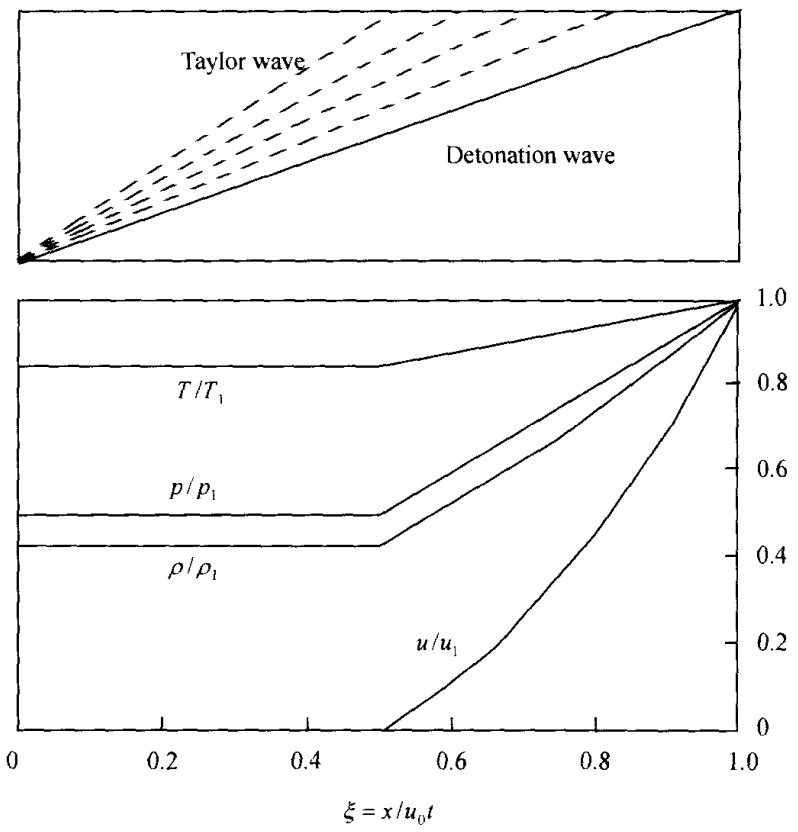

Fig. 1. The gas state distributions following the detonation wave. 
homogenous zone. At any time, the length of this zone is about half of the propagation distance of the detonation wave.

\subsection{Characteristics of the backward detonation driver}

The detonation wave propagates upstream in the backward detonation driver. The wave diagram of flow is depicted in fig. 2 . The backward detonation driver induces additional detonation waves, as well as following Taylor wave $R_{\mathrm{T}}$, as compared with the wave diagram of a calssical shock tube. The Taylor wave tail is in parallel with the wave head of the central rarefaction wave generated after the rupture of diaphragm. The characteristics of the backward detonation driver are attributed to the state of the gas in zone 4. As noted previously, the gas is stationary and the thermodynamic-state parameters are homogenous in zone 4 . Therefore, the extra detonation waves and Taylor wave have no effect on the flow in the shock tube before the reflected waves (which are formed from reflection of the detonation wave on the rear wall of the driver section) overtake them. The driving characteristics of the backward detonation correspond with that of the classical shock tube with a high-pressure gas driver. The driving capability of the backward detonation is stronger because the acoustis velocity is higher. Experimental results have confirmed the expectations ${ }^{[12]}$.

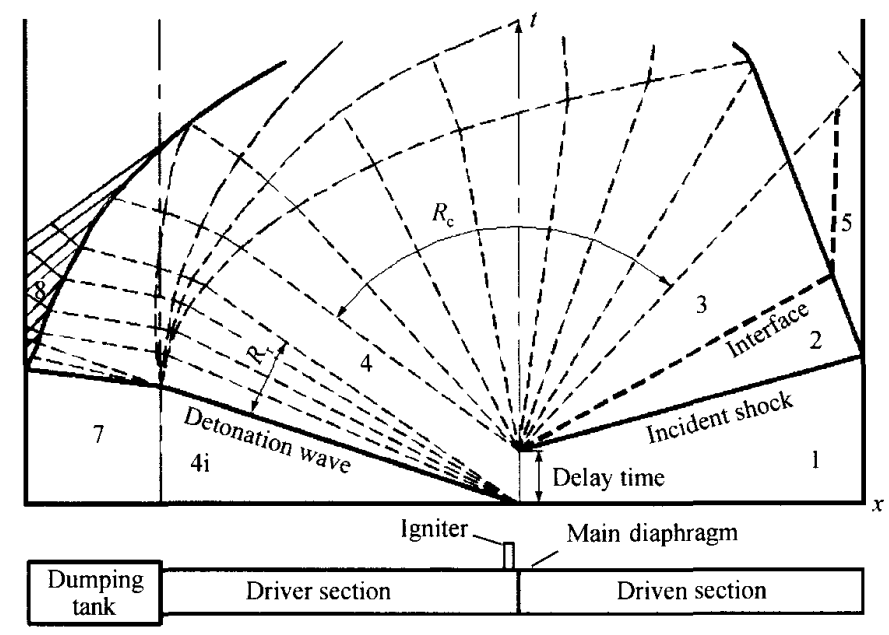

Fig. 2. Wave diagram of backward detonation driver.

\subsection{Characteristics of the forward detonation driver}

The detonation wave propagates downstream in a forward detonation driver. The wave diagram in fig. 3 indicates that the Taylor wave behind the detonation wave mutually interferes with the central rarefaction wave. The driving gas parameters in the wave head of the central rarefaction wave (such as speed, temperature, pressure, etc.) decline over time. This declining means that the effective driving capability is weakened gradu- 
ally. Penetrating the central rarefaction waves, the Taylor wave overtakes the incident shock wave and reduces its intensity continuously. Thus, it is hard to achieve a steady testing gas by the forward detonation driver.

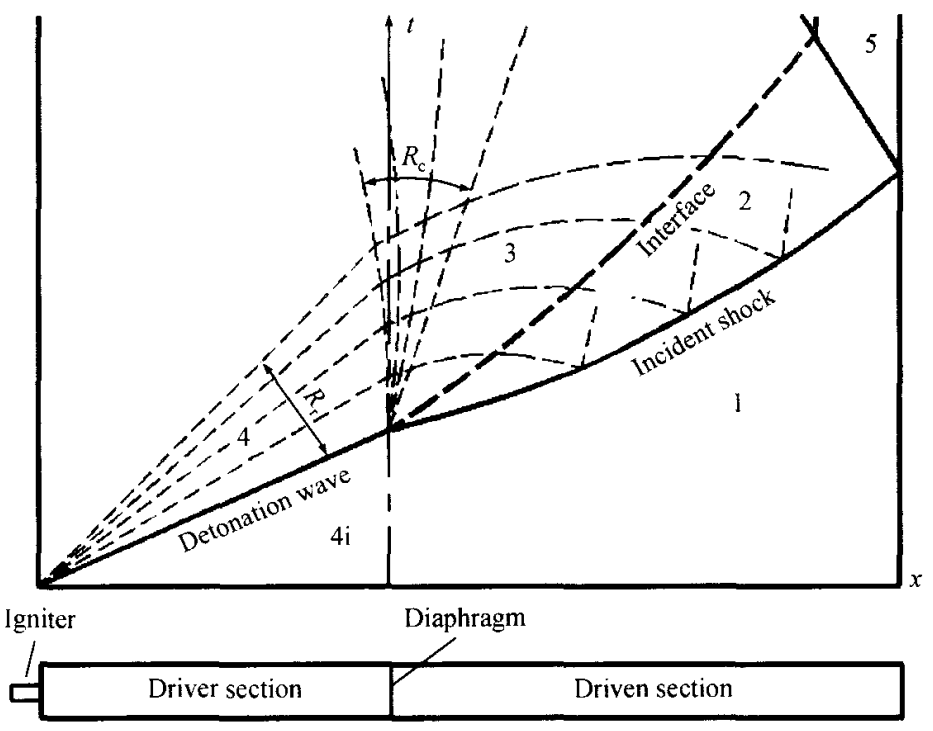

Fig. 3. Wave diagram of forward detonation driver.

1.4 Comparison of the characteristics between backward and forward detonation drivers

As to driving quality, the effective driving conditions of the backward detonation driver are uniform, whereas those of the forward detonation driver attenuate with the time. The former is better than the latter in driving quality.

As to driving capability, the effective driving gas parameters of the backward detonation driver are the parameters in the stationary zone behind the Taylor wave. Both pressure and temperature are much lower than the $\mathrm{CJ}$ value of the detonation product. The pressure and temperature of the forward detonation driving gas vary with time, and their values are higher than those of the backward detonation driver. In addition, the driving capability of the forward detonation is further promoted owing to the tremendous kinetic energy of the detonation product flowing forward. In conclusion, the driving capability of the forward detonation is much higher than that of the backward detonation.

Using the same initial conditions (detonation gas: $90 \% \mathrm{H}_{2}+10 \% \mathrm{O}_{2}$, initial pressure $20 \mathrm{MPa}$; driver gas = air with initial pressure $0.1 \mathrm{MPa}$; both initial temperatures = $15^{\circ} \mathrm{C}$ ) and assumptions (the wall influence was ignored; the specific heat ratio of all gas was 1.4 for the full process), Bird ${ }^{[13]}$ calculated that the maximum Mach number Ms of the incidence shock wave generated in the backward detonation driver is 9.8 and in the 
forward detonation driver is 15.8 . Yu's experimental results ${ }^{[12]}$ indicated that the initial pressure of the detonation gas required in the forward detonation driver is lower by one order of magnitude than that in the backward detonation driver when the initial pressures in the driven sections and the maximal Mach numbers are identical respectively in both the detonation drivers. Thus, the conclusions above, that the driving capability of the forward detonation is stronger, were confirmed once again.

\section{Method of eliminating the Taylor rarefaction wave}

If a moving piston is following a detonation wave, the gas velocity of the Taylor wave tail after the detonation wave is equal to that of the piston. If the velocity of the piston reaches that of the detonation gas $\left(u_{\mathrm{P}}=u_{\mathrm{CJ}}\right)$, a Taylor wave will not occur. Moreover, what follows the detonation wave will be an expanding gas column with constant velocity and state parameters. Under the condition that the velocity of the piston exceeds that of the detonation gas $\left(u_{\mathrm{P}}>u_{\mathrm{CJ}}\right)$, a Taylor wave will not occur, as is for the case of $u_{\mathrm{P}}$ $=u_{\mathrm{CJ}}$. The propagation velocity of the detonation wave driven by the piston with higher velocity is faster and the gas-state parameters after detonation are higher.

Because the gas velocity behind a detonation wave is very high $(>1 \mathrm{~km} / \mathrm{s})$, it is difficult to accelerate a mechanical piston to such a high speed in shock tube. Coates et al. ${ }^{[6]}$ employed an additional driver utilizing high-pressure hydrogen as the driving gas attached to the upstream end of the detonation driver, which generated a moving gas column instead of a traveling high-speed piston. The surface pressure of the gas column must be the same as that of the contact plane with the detonation gases. For equating the velocity of the gas column to that of the detonated gas, the initial pressure of hydrogen should be at least 100 times higher than that of the hydrogen-oxygen mixed gases in the detonation section. Bakos et al. ${ }^{[14]}$ took helium as the driving gas in the additional driver. Under such conditions, the pressure ratio of the helium to the hydrogen-oxygen mixture is in need of more times. The initial pressure of mixed gases in the detonation driver, where the testing gases with high enthalpy and high pressure are generated, is most often $10 \mathrm{MPa}$ or higher. Therefore, the required initial pressure of hydrogen or helium must be excessively high in order to eliminate the Taylor wave completely in the forward detonation driver. Not only are the required high-pressure pump and vessel equipment too expensive, but also the structures of the auxiliary driver as well as the rupture technology of diaphragm present severe technical problems.

For solving these technical problems, $\mathrm{Yu}^{[8]}$ proposed to utilize hydrogen-oxygen mixed gases instead of light gases as the driving gas in the additional driving section. Under these conditions, the initial pressure of the additional detonation driver needs only be several times higher than that of the primary section to eliminate the Taylor wave, and the thermodynamic parameters as well as the velocity of driving gases will be homogenous. In addition, the detonation gas ejected from the additonal driving section can directly initiate a detonation wave in the primary driving section. Thus, another technical impediment is also solved in practice. 


\subsection{Effect of the ratio of initial pressures in additional driver and primary driver}

The wave diagram of a shock tube with double detonation drivers are shown in fig. 4. When the initial pressure ratio of the additional driver to the primary driver $\left(P_{8 \mathrm{i}} / P_{4 \mathrm{i}}\right)$ is low, the velocity of the detonated gases accelerated by the expansion in the additional driver is still lower than that of the detonation gas in the primary driver. The Taylor wave is therefore eliminated partially, the angle of the wave fan decreases as compared with that at the beginning detonation of the close section (shown in fig. 4(a)). If the initial pressure ratio is close to the critical value, the velocity of the detonated gases accelerated by the expansion is equal to that of the detonation gases, and thus the Taylor wave is eliminated completely (shown in fig. 4(b)). If the initial pressure ratio is higher than the critical value, the diagram depicted in fig. 4(b) still applies, whereas, the detonation

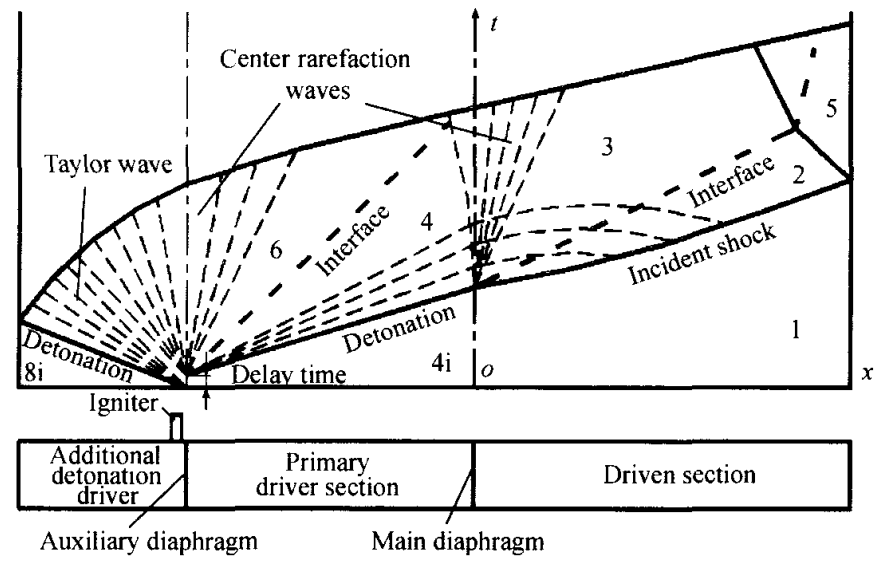

(a)

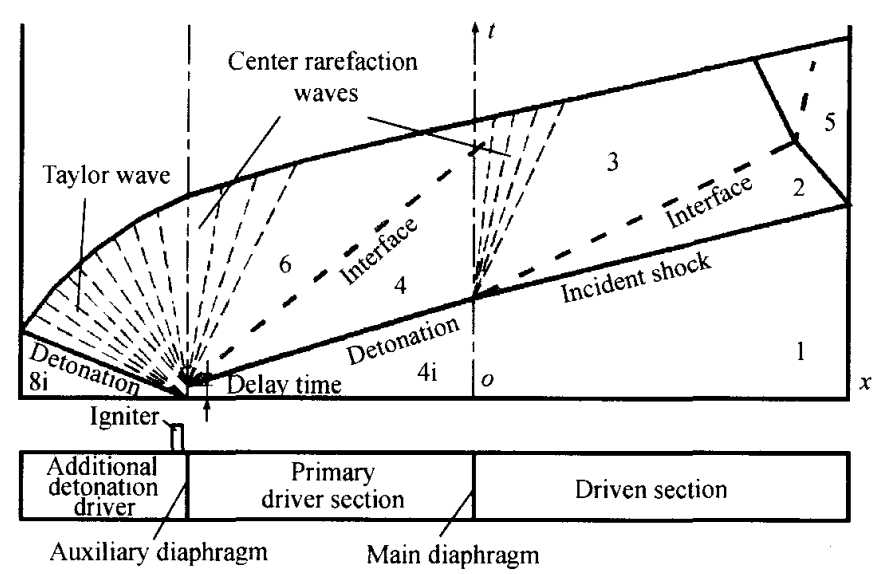

(b)

Fig. 4. Wave diagram of shock tube with double detonation drivers. (a) $P_{8 \mathrm{i}} / P_{4 \mathrm{i}}<$ the critical value; (b) $P_{8 \mathrm{i}} / P_{4 \mathrm{i}} \geqslant$ the critical value. 
waves in the primary driver are transformed into an over-driving detonation wave, the velocity of which is higher than that of a CJ detonation wave. The temperature, pressure, and velocity of detonation gases rise with each increment of $P_{8 \mathrm{i}} / P_{4 \mathrm{i}}$ value. Therefore, increasing the initial pressure in the additional section is an effective way to further intensify the driving capability of the forward detonation section.

\subsection{Estimation of the ratio of the critical initial pressure}

Penetrating the Taylor wave, the detonated gases in the additional driving section are decelerated to the stationary state, and then accelerated to the $u_{6}$ after penetrating the central rarefaction wave.

$$
u_{8 \mathrm{CJ}}+\frac{2}{\gamma-1} a_{8 \mathrm{CJ}}=u_{6}+\frac{2}{\gamma-1} a_{6} .
$$

On the assumptions (1) that the ratio of the specific heat of detonation gases in the expansion process is constant $(\gamma=1.2)$, (2) that the component ratio of hydrogen to oxygen is 3 , (3) that the initial temperature of the mixed gases is $18^{\circ} \mathrm{C}$ in the additional/primary drivers, (4) that the initial pressure has no influence on the gas parameters and the pressure ratio of the wave front to wave rear, (5) that values taken from ref. [4] $\left(u_{\mathrm{CJ}}=1443 \mathrm{~m} / \mathrm{s}\right.$ and $\left.a_{\mathrm{CJ}}=1845 \mathrm{~m} / \mathrm{s}\right)$ are applicable, and (6) that the Taylor wave is eliminated behind the detonation wave, and we obtained $P_{6}=P_{4}=P_{4 \mathrm{CJ}}, u_{6}=u_{4}=u_{4 \mathrm{CJ}}=$ $-u_{8 \mathrm{CJ}}$, and then $a_{8 \mathrm{CJ}} / a_{6} \approx 1.18$ and $P_{8 \mathrm{CJ}} / P_{6} \approx 7$. Thus, there exists a relation $P_{6}=P_{4}=P_{4 \mathrm{CJ}}$ and then,

$$
\left(P_{8 \mathrm{i}} / P_{4 \mathrm{i}}\right)_{\mathrm{CR}} \approx P_{8 \mathrm{CJ}} / P_{4 \mathrm{CJ}} \approx 7
$$

\section{Experimental results}

In order to observe the gas parameters behind the detonation wave varying with time in the primary detonation driver, pressure sensors were installed at 0.45 and $1.95 \mathrm{~m}$ from the diaphragm between the additional and the primary detonation drivers. The measured results are depicted in fig. 5, in which results of the forward detonation driver without the additional driver are shown in fig. 5(a) for comparison. Behind the detonation wave, the abrupt pressure increased to be seventeen times higher than the initial pressure (fig. 5(a)). Subsequently, the pressure dropped below half of the peak value, owing to the effect of the Taylor wave, and then maintained a constant value. The pressure of the tail of the curve, measured at $1.95 \mathrm{~m}$, decreased again because the central rarefaction wave generated after rupture of the main diaphragm overtook and attenuated the detonation wave. A plot of the pressure for the condition of $P 8 \mathrm{i} / P_{4 \mathrm{i}}=4$ is given in fig. 5(b). Because the initial pressure ratio was lower than the critical value, the pressure rose initially and then dropped. However, the period of the drop was shorter and the pressure platform value was higher than the results shown in fig. 5(a). Fig. 5(c) reveals the pressure curve for the condition of $P_{8 \mathrm{i}} / P_{4 \mathrm{i}}=6$. Because the initial pressure ratio was close to the critical value, the pressure rose, but then did not drop. This response indi- 


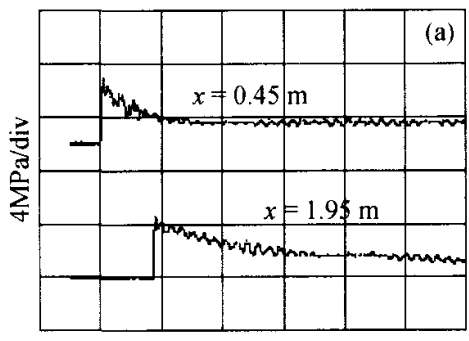

$0.5 \mathrm{~ms} / \mathrm{div}$

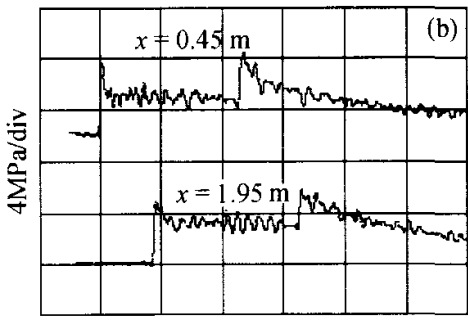

$0.5 \mathrm{~ms} / \mathrm{div}$

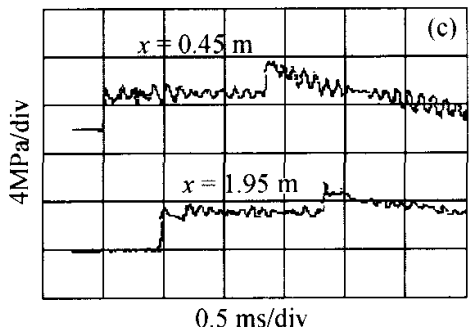

Fig. 5. Pressure-time curves at the primary driver section. (a) Single detonation section $\left(P_{4 \mathrm{i}}=0.25 \mathrm{MPa}\right)$; (b) double detonation section $\left(P_{8 \mathrm{i}} / P_{4 \mathrm{i}}=4, P_{4 \mathrm{i}}=0.25 \mathrm{MPa}\right)$; (c) double detonation section $\left(P_{8 \mathrm{i}} / P_{4 \mathrm{i}}=6, P_{4 \mathrm{i}}=0.2 \mathrm{MPa}\right)$.

cates that the Taylor wave was eliminated. The pressure platform value was equal to the peak value. Because the reflected waves that formed from reflection of the backward detonation wave at the tail-end wall of the driver section overtook the detonation wave and caused interference, the pressure of the curve tail had an upward tendency. Such interference will not result in the disadvantageous influence on the flow in the shock tube if the length of each section is designed properly.

The intensity variations of the incident shock waves generated in double detonation drivers and propagating along the driven section and those caused in the single forward detonation driver are compared in fig. 6. Under the condition that the initial pressures in the driven sections and the maximal Mach numbers are identical respectively, the attenuation ratio of the Mach number of an incident shock wave generated in the double detonation driver is lower than that in the single forward detonation driver. However, the attenuation ratio of the Mach number of the incident shock wave was appreciable, even for a wave generated in the double detonation driver because the attenuation was dependent not only on the quality of the driving gases, but also on the boundary layer of the tube wall in the driven section. For the apparatus used in our work, the surface state of the tube wall was not ideal and the initial pressure was lower; inevitably, boundary-layer effects induced attenuation of the shock wave. In practical applications, the attenuation of the incident shock wave in the double detonation driver will be reduced significantly if we improve the state of the tube wall and increase the initial pressure. Then, the influence of the boundary layer effect will decrease substantially. 


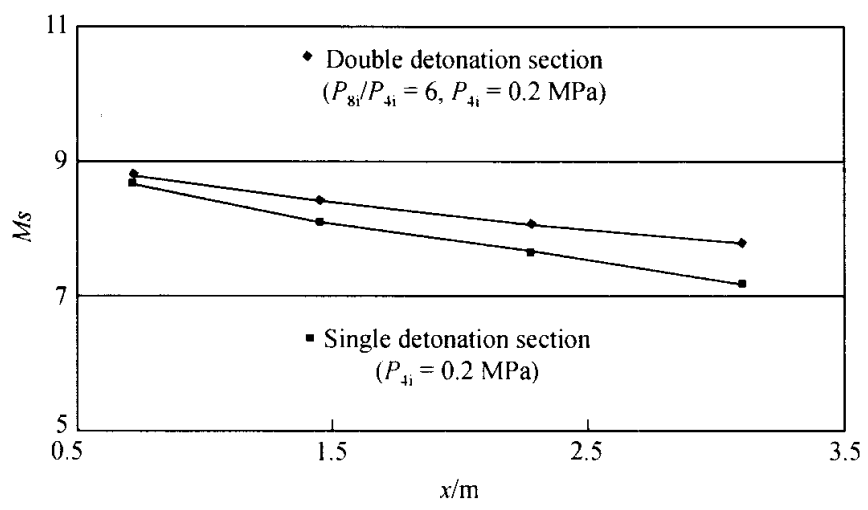

Fig. 6. Attenuation characteristics of incident shock wave.

\section{Conclusion}

The driving capability of a forward detonation driver is more powerful than that of a backward denotation driver. Because parameters of the driving gases vary with time, the incident shock wave generated is attenuated heavily so that requirements of practical utilization are difficult to attain. It was concluded that the backward detonation driver is connected to the upstream end of the detonation driver. If the ratio of the initial pressure in the additional section to the primary section is greater than the critical value, the Taylor wave will be eliminated and driving gases of fine quality can be generated. Double detonation drivers have the possibility of being the applicable strong drivers, which can generate testing gases of high enthalpy and high stationary pressure.

In addition, an over-driving detonation wave will be obtained by further increasing the ratio of the initial pressure in the additional section to that in the primary section. Its driving capability will be further strengthened. Consequently, it constitutes a new way to improve the enthalpy value of testing gases in shock tubes/tunnels.

Acknowledgements This work was supported by the National Natural Science Foundation of China (Grant No. 10082004).

\section{References}

1. Yu, H. R., Shock tunnel and its application to aero-heating experiments (in Chinese), Thesis, Institute of Mechanics, Chinese Academy of Sciences (1963).

2. Lee, B. H. K., Detonation driven shocks in a shock tube, AIAA J., 1967, 5: 791—792.

3. Gier, H. L., Jones, T. G., An investigation of a double diaphragm shock tube with a detonating buffer gas, in Shock Tubes (ed. Glass, II), Toronto: University of Toronto Press, 1970, 272-290.

4. Edwards, D. H., Willians, G. T., Breeze, J. C., Pressure and velocity measurements on detonation waves in hydro-oxygen mixtures, Fluid Mechanics J., 1959(6): 497-517.

5. Balcarzak, M. J., Johnson, M. R., The gaseous detonation driver and its application to shock tube simulation techniques, in Proceeding $5^{\text {th }}$ Int. Symp on Shock Tubes (eds. Moulton, J. F., Filler, W. S.), White Oak, Maryland USA, American Physical Society (AD638011), 1965, 1111-1119. 
6. Coates, P. B., Gaydon, A. G., A simple shock tube with detonating driver gas, Proc. Roy. Soc., London, 1965, A283:18-32.

7. Yu, H. R., Recent developments in shock tube application, Proc. of the 1989 Japan National Symposium, On shock wave phenomena, Sagamihara, Tohoku Uni press Seudai, Japan, 1989, 1-7.

8. Yu, H. R., Esser, B., Lenartz, M. et al., Gaseous detonation driver for a shock tunnel, Shock Waves, 1992, 2(4): $245-254$.

9. Bakos, R. J., Erdos, J. I., Options for enhancement of the performance of shock-expansion tubes and tunnels, AIAA, 1995, p95-0799.

10. Taylor, G. I., The dynamics of the combustion products behind planar and spherical detonation fronts in explosive, Proc. Roy. Soc., 1950, A200: 235-247.

11. Zel'dovich, Y. B., Distribution of pressure and velocity in detonation products, J. Exp. and Theor. Physics, 1942, 12: 389.

12. Yu, H. R., Oxy-hydrogen combustion and detonation driven shock tube, Acta Mechanica Sinica, 1999,15(2): $97-107$.

13. Bird, G. A., A note on combustion driven shock tubes, RAE TN-Aero 2511, 1957, Farnborough, England.

14. Bakos, R. J., Erdos, J. I., An experimental and computational study leading to new test capabilities for the hypulse facility with a detonation driver, AIAA, 1996, p96-2193: 17-20. 